\title{
How do investors' expectations drive asset prices?
}

\author{
Erik Lüders*, Bernhard Peisl ${ }^{\dagger}$ \\ March 2001
}

\begin{abstract}
Asset price processes are completely described by information processes and investors' preferences. In this paper we derive the relationship between the process of investors' expectations of the terminal stock price and asset prices in a general continuous time pricing kernel framework. To derive the asset price process we make use of the modern technique of forward-backward stochastic differential equations. With this approach it is possible to show the driving factors for stochastic volatility of asset prices and to give theoretical arguments for empirically well documented facts. We show that stylized facts that look at first hand like financial market anomalies may be explained by an information process with stochastic volatility.
\end{abstract}

JEL Classification: C69, G12

Keywords and Phrases: backward stochastic differential equations, information processes, pricing kernel

\footnotetext{
${ }^{*}$ Center of Finance and Econometrics, University of Konstanz and Zentrum für Europäische Wirtschaftsforschung (ZEW), Mannheim

Email: Erik.Lueders@uni-konstanz.de

${ }^{\dagger}$ Center of Finance and Econometrics, University of Konstanz

Email: Bernhard.Peisl@uni-konstanz.de
} 


\section{Introduction}

In the last decades much empirical work has been done on time series of asset prices. Many studies report mean reversion in stock returns [see Fama and French [9]; Poterba, Summers [28]], predictability of the equity premium and other "anomalies". Empirical research on options suggests significant mispricing compared to theoretical option prices, especially compared to the Black-Scholes model (see Canina, Figlewski [4]; Jackwerth, Rubinstein [20]; Rubinstein [29] and for an outstanding survey Ghysels, Harvey, Renault [13]). Most of these well documented facts still lack a sound theoretical explanation. While the smile effect, for example, can be explained with stochastic volatility models the stochastic process of the volatility is usually exogeneously given. Only few models address the basic economic question why volatility is stochastic. Thus, usually a somewhat arbitrary volatility process is introduced. ${ }^{1}$

Many theoretical papers have already investigated the viability of stochastic processes for asset prices, i.e. the consistency with an equilibrium. A common framework for the investigation is a representative investor economy. Basically, as was shown for example by Decamps, Lazrak [6] an equilibrium in a representative investor economy implies that the pricing kernel is a deterministic function of wealth. Hence, not every arbitrage free asset price process is consistent with such an equilibrium, since the existence of a strict positive pricing kernel is sufficient to ensure the absence of arbitrage possibilities. The assumption of arbitrage free markets does not imply that the pricing kernel is a deterministic function of wealth. Whether certain asset price processes are consistent with an equilibrium in a representative investor economy has been analyzed for example in Bick [1]; Bick [2]; He, Leland [14] and Pham, Touzi [27]. Franke, Stapleton, Subrahmanyam [12] choose a slightly different, more constructive approach to investigate the viability of asset price processes. Instead of starting with the stochastic process of asset prices, they take the process of investors' expectations of the future cash flow as given. They further assume that the asset pays no dividends. Since the price is completely described by the distribution of the cash flow and by investors' preferences it is possible to construct any viable asset price process from the characteristics of information processes and preferences. By

\footnotetext{
[32].

${ }^{1}$ For models that are able to generate stochastic volatility see David [5] and Veronesi
} 
the assumption of rational investors it is possible to impose restrictions on the process representing investors' expectations, i.e. the information process. Hence, with the information process the distribution of the cash flow is given and from the assumptions on investors' preferences the characteristics of the pricing kernel are given, too. Thus, the asset price process can be derived from the underlying assumptions.

In this paper we follow the approach of Franke, Stapleton, Subrahmanyam. While Franke, Stapleton, Subrahmanyam emphasize the importance of the utility function or more precisely the elasticity of the pricing kernel our task is to show the influence of the variations in expectations, i.e. the influence of the volatility of the information process on the asset price process. We extend their approach in that we allow for a second risk factor driving the process of investors' expectations. Hence, in our model the volatility of the information process may be stochastic. Further we will give an economic justification for the generalization. We are arguing that introducing stochastic volatility of the information process is a sensible assumption. To see this consider a stochastic process with only one risk factor, e.g. the geometric Brownian motion. In this case, the uncertainty about the stock price in $T$ is an only time dependent deterministic function. It is sensible to assume, that this uncertainty may also be a stochastic function since this uncertainty is driven by exogenous shocks. Unexpected news announcements may be seen as one of these exogenous shocks. We will turn to this point again in section 3 .

With our approach we are able to link explicitly financial markets phenomena to the process of investors' expectations. We will show that many properties of asset price processes and especially empirically documented properties of the risk premia can be explained by the characteristics of the volatility of the information process. Further, we give an economic justification for stochastic volatility asset models and we discuss the justification of specifications of stochastic volatility by relating them to the process of investors' expectations.

The organization of this paper is as follows. The next section gives a short review on related papers. In section 3 we discuss the viability of information processes under the assumption of rational expectations. In section 4 we give a brief characterization of the pricing kernel. In section 5 we derive viable asset price processes with the modern technique of forward-backward stochastic differential equations (an outstanding overview on backward stochastic differential equations and forward-backward stochastic differential equations is 
given in El Karoui et al. [8]). Section 6 summarizes the main results.

\section{A Short Survey on Related Papers}

Before the seminal paper of Huang [19] continuous time models in finance were already prevalent. The usual assumption was that the equilibrium asset prices can be represented by Itô integrals. Huang was the first to give a sound theoretical justification for this assumption. One main result in his paper is that if equilibrium asset prices are adapted to a filtration generated by a Brownian motion, then equilibrium asset prices are Itô integrals. Thus Huang provided a justification for continuous sample paths of equilibrium asset prices by linking them to the information flow.

The studies in the 90's on the foundation of equilibrium asset price processes addressed the question which characteristics of the state price density can be supported by sensible assumptions on the utility function of a representative agent. Connected to this was the question, which utility functions are implied by equilibrium asset prices which are governed by specific stochastic differential equations. Bick [2] characterizes processes as viable by the "no-trade criteria", i.e. an asset price process is a possible equilibrium if there exists a von Neumann-Morgenstern utility function such that it is optimal for the representative agent to buy the market portfolio in $t=0$ and hold it until $T$. Bick requires path-independence of the pricing kernel for viability which is equivalent to the requirement that the pricing kernel is a deterministic function of wealth. ${ }^{2}$ Ensuing papers of He, Leland [14], Hodges, Carverhill [17], Hodges, Selby [16] and Decamps, Lazrak [6] generalize this analysis further.

Pham and Touzi [27] tackle the case of stochastic volatility. They provide utility-theoretic foundations for common assumptions on the risk premia in stochastic volatility models. Their analysis is similar to the previously mentioned, as they start with stochastic differential equations for the asset prices. The main results of their paper are necessary and sufficient conditions for the viability of the risk premia. Of special interest may be their analysis of the classical stochastic volatility model of Hull and White [18] and the concept of a minimal martingale measure introduced by Föllmer and Schweizer [10]. Hull and White were the first to derive an explicit formula for the price of an European option written on an asset with stochastic volatility. Yet their

\footnotetext{
${ }^{2}$ See for example Decamps, Lazrak [6] and section 4 of this paper.
} 
result crucially depends on the assumption that the volatility risk premium is independent of the underlying asset. Since in an incomplete market the equivalent martingale measure and, thus, the risk premia are not uniquely determined by arbitrage arguments, some restriction has to be imposed on the risk premia. The analysis of Pham and Touzi establishes that this kind of volatility risk premium is consistent with constant relative risk aversion. For the specification of the equivalent martingale measure in incomplete markets Föllmer and Schweizer [10] propose the concept of a minimal martingale measure. Loosely stated the minimal martingale measure is defined such that only traded risk is priced, hence, risk that is uncorrelated with traded assets has a price of zero. As intuition suggests this kind of equilibrium is supported by logarithmic preferences.

The analysis of Franke, Stapleton and Subrahmanyam [12] differs in various ways from the former papers. First, they do not assume the existence of a representative investor, instead they simply assume that markets do not admit arbitrage possibilities and hence, a pricing kernel exists. Second, they do not take the asset price process as given. Their approach is more constructive as the basis of the model is a process for conditional expectations of the exogenously given asset price at some terminal date. From the assumption of rational investors they deduce the martingale property of the process of conditional expectations. With the assumption that the process of conditional expectations is governed by a geometric Brownian motion without drift their analysis establishes a strong relationship between the process of conditional expectations and the asset price process. In particular, they show that the asset price process follows a geometric Brownian motion if conditional expectations follow a geometric Brownian motion without drift and the pricing kernel has constant elasticity. They also derive properties of the price process for a pricing kernel with either declining or increasing elasticity. In these cases asset returns are autocorrelated and the variance of the asset price is higher than with constant elasticity of the pricing kernel.

\section{Characterization and Viability of Informa- tion Processes}

In this section we introduce an information process $I$ similar to the one in Franke, Stapleton, Subrahmanyam [12]. It is defined as the process of 
conditional expectations of investors about a future cash flow. Thus, this process describes all the information available to the investors. We focus on the question how the asset price process is depending on the characteristics of such an information process $I$ while we take the information process as given.

We consider a market with the given time horizon $T>0$ and the two dimensional standard Brownian motion $W=\left\{\left(W_{t}^{I}, W_{t}^{V}\right): t \in[0, T]\right\}$ on a given probability space $\left(\Omega, \mathcal{F}, \mathcal{F}_{t}, P\right)$ where $\left(\mathcal{F}_{t}\right)_{t \in[0, T]}$ is the filtration generated by $W$ augmented by all the $\mathcal{F}$-null sets, with $\mathcal{F}=\mathcal{F}_{T}$. The information process $I$ is defined on this probability space. As already mentioned, this process is assumed to represent investors' expectations about the future cash flow paid to the shareholder of a company. We assume that this strictly positive dividend is paid only in $T$. Since investors are assumed to act totally rational, $I_{t}$ is a positive $P$-martingale and hence admits the following representation ${ }^{3}$

$$
I_{t}=I_{0}+\int_{0}^{t} I_{s}\left(\sigma_{s}^{I} d W_{s}^{I}+\sigma_{s}^{I, V} d W_{s}^{V}\right), \quad 0 \leq t \leq T .
$$

The martingale representation theorem provides that there exist two processes $\sigma_{t}^{I}$ and $\sigma_{t}^{I, V}$. By this theorem one only knows that these processes are adapted to $\mathcal{F}_{t}$ and that $P\left(\int_{0}^{T}\left(\sigma_{s}^{I}\right)^{2} d s<\infty\right)=1$ and $P\left(\int_{0}^{T}\left(\sigma_{s}^{I, V}\right)^{2} d s<\infty\right)=1$. In the following we make the assumption $\sigma_{t}^{I, V}=0$ for all $t \in[0, T]$ and we require a special characterization of $\sigma_{t}^{I}$. Of course, with these assumptions we assume a special representation of $I_{t}$. Later we will give some economic arguments for this special representation.

In the remainder of this paper we assume that the volatility $\sigma_{t}^{I}$ of the information process is governed by the following stochastic differential equation:

$$
\sigma_{t}^{I}=\sigma_{0}^{I}+\int_{0}^{t} b\left(s, I_{s}, \sigma_{s}^{I}\right) d s+\int_{0}^{t} \sigma^{V}\left(s, I_{s}, \sigma_{s}^{I}\right) d W_{s}^{V}, \quad 0 \leq t \leq T,
$$

where $b$ is the drift and $\sigma^{V}$ describes the volatility of $\sigma_{t}^{I}$ (these two functions are assumed to be deterministic). Since $I_{t}$ represents investors' expectations in $t$ about the value of the asset in $T$, the process $I_{t}$ and the forward

\footnotetext{
${ }^{3}$ See for example Karatzas and Shreve [22].
} 
price process of the asset $F_{t}$ must be equal at time $T$. Thus, by definition $F_{T}=I_{T}$. Hence, by postulating such an information process $I_{t}$ the value of the asset in $T$ which is equal to the cash flow at date $T$ is given. Equation (1) is a generalization of the information process considered in Franke, Stapleton, Subrahmanyam [12]. While their information process is modeled as a geometric Brownian motion, equation (1) admits constant, time varying, deterministic or stochastic volatility.

In the remainder of this paper $\sigma\left\{I_{u} \mid 0 \leq u \leq t\right\}$ represents the filtration generated by $I_{t}$ which by assumption represents all the information available to investors in $t$. We will now turn to the economic meaning of different volatility models. First take the case of constant volatility, i.e. $\sigma_{t}^{I}=\sigma_{0}^{I}$ for all $t \in[0, T]$. Hence, the logarithm of $I_{T}$ is normally distributed with expectation $E^{P}\left(\ln I_{T} \mid \sigma\left\{I_{u} \mid 0 \leq u \leq t\right\}\right)=\ln \left(I_{t}\right)-\frac{\left(\sigma_{0}^{I}\right)^{2}}{2}(T-t)$ and variance $\operatorname{var}\left(\ln I_{T} \mid \sigma\left\{I_{u} \mid 0 \leq u \leq t\right\}\right)=\left(\sigma_{0}^{I}\right)^{2}(T-t)$ for $0 \leq t \leq T$. Since the price of the asset in $T$ is equal to $I_{T}$ this implies that the uncertainty about the final value of the asset is a linearly decreasing function of time.

This constant rate of uncertainty resolution over time implies some special information flow. The intensity of information arrival must be constant over time, i.e. there are no periods where more information is getting into the market than during other periods. Deterministic but time varying volatility would allow for periods with a more intense information flow, but uncertainty resolution is still a deterministic function of time. Such deterministic time patterns might be explained by some sort of clustering of the information flow; companies announcing their results in certain periods, e.g. at the end of a year, many macroeconomic announcements such as monthly economic information releases occur at certain week-days (see Ederington and Lee [7] for a related econometric study). These facts may explain to some degree a deterministic time pattern of the volatility of the information process. Hence, under the assumption of time varying volatility the conditional variance of $I_{T}$ is no more linear in $t$. But the volatility and the process of conditional variances of $I_{T}$ can still be perfectly forecasted.

Since the information process is governed by such scheduled information releases but also by unforeseen information events we consider some randomness in the volatility of the information process to get a more realistic model for the conditional expectations. First assume that volatility is a borel 
function of $t, I_{t}$ and $\sigma_{t}^{I}$, hence it is a deterministic function of $t, I_{t}$ and $\sigma_{t}^{I}$ :

$$
\sigma_{t}^{I}=\sigma_{0}^{I}+\int_{0}^{t} b\left(s, I_{s}, \sigma_{s}^{I}\right) d s, \quad 0 \leq t \leq T .
$$

This formulation of the volatility is, for example, consistent with a leverage effect, i.e. volatility increases with decreasing asset value. The easiest way to model the leverage effect, is to assume a constant elasticity of variance model (CEV)

$$
\sigma_{t}^{I}=\bar{\sigma} I_{t}^{-\alpha}, 0 \leq t \leq T \text {, for some } \alpha \text { with } 0 \leq \alpha \leq 1 \text { and } \bar{\sigma}>0 \text { constant. }
$$

The model (equation (3)) includes all variations of the volatility of the information process which can be described by deterministic functions of $t$, $I_{t}$ and $\sigma_{t}^{I}$. Notice that with our model (equation (3)) $\sigma_{t}^{I}$ is random since it is a function of $I_{t}$, but it is $\sigma\left\{I_{u} \mid 0 \leq u \leq t\right\}$-measurable, thus the current volatility is known. Economically this means that the current (short-term or myopic) risk is known but the long-term risk evolves stochastically over time.

Even this more general model neglects some kind of uncertainty. Many news about the economy or politics as well as about markets and companies are published completely erratically so that stochastic terms have to be considered explicitly in the volatility process. Therefore to include these facts in the model, the volatility is governed by a separate stochastic differential equation with a stochastic term (equation (2)). It will be obvious from Theorem 1 that modeling the volatility of the information process by a separate stochastic differential equation with a stochastic term has an important effect on the asset price process. With this model, the volatility risk of the information process is priced, i.e. a risk premium is paid. Hence, all other variations in the volatility of the information process are not priced.

We can conclude that in general we have to consider stochastic volatility which exhibits some time pattern. The quantification of these facts is an empirical task and is closely related to the estimation of the volatility of asset prices.

\section{Characterization of the Pricing Kernel}

To derive the asset price process we need the Girsanov-theorem. In this section we give a brief summary of Girsanov's theorem and an economic 
interpretation of the process defining the pricing kernel. Further, we apply Girsanov's theorem in a representative investor economy to gain a deeper understanding of the pricing kernel.

\subsection{Brief summary of Girsanov's theorem}

Assuming that the market, in some filtered probability space $\left(\Omega, \mathcal{G}, \mathcal{G}_{t}, P\right)$ on which defined a standard Brownian motion $W$, admits no arbitrage possibilities it is well known that there exists a martingale measure $\widehat{P}$ equivalent to $P$ under which the forward price $F_{t}$ is a martingale ${ }^{4}$, thus

$$
F_{t}=E^{\widehat{P}}\left(F_{T} \mid \mathcal{G}_{t}\right), \quad 0 \leqslant t \leqslant T .
$$

Defining the density process $\eta_{t}:=E^{P}\left(\frac{d \widehat{P}}{d P} \mid \mathcal{G}_{t}\right), 0 \leqslant t \leqslant T$, then $F$ follows a $\widehat{P}$-martingale if and only if $\eta F$ follows a $P$-martingale, in other words

$$
\eta_{t} F_{t}=E^{P}\left(\eta_{T} F_{T} \mid \mathcal{G}_{t}\right), \quad 0 \leqslant t \leqslant T .
$$

Now, for an adapted real-valued process $\lambda$ with $P\left(\int_{0}^{T} \lambda_{t}^{2} d t<\infty\right)=1$ consider

$$
\begin{aligned}
d \Phi_{t}^{(\lambda)} & =-\Phi_{t}^{(\lambda)} \lambda_{t} d W_{t}, \quad 0 \leqslant t \leqslant T, \\
\Phi_{0}^{(\lambda)} & =1,
\end{aligned}
$$

then it is clear that $\Phi^{(\lambda)}$ is a $P$-martingale. Further, the unique solution to equation (4) is $\Phi_{t}^{(\lambda)}=\exp \left(-\int_{0}^{t} \lambda_{u} d W_{u}-\frac{1}{2} \int_{0}^{t}\left|\lambda_{u}\right|^{2} d u\right)$ for $0 \leqslant t \leqslant T$. By construction the process $\Phi^{(\lambda)}$ has the properties of a density process.

However, it follows from the no arbitrage assumption that there exists a process $\kappa$ such that $\Phi^{(\kappa)}$ defines a probability measure $\widetilde{P}$ by

$$
\frac{d \widetilde{P}}{d P}=\Phi_{t}^{(\kappa)}, \quad 0 \leqslant t \leqslant T, P \text {-a.s. }
$$

under which $F$ is a martingale. Hence, we have

$$
F_{t}=E^{P}\left(\Phi_{t, T}^{(\kappa)} F_{T} \mid \mathcal{G}_{t}\right), \quad 0 \leqslant t \leqslant T,
$$

\footnotetext{
${ }^{4}$ We consider only forward contracts with maturity time $T$, thus for notational convenience we write $F_{t}$ instead of $F_{t, T}$.
} 
where for notational convenience we write $\Phi_{t, T}^{(\kappa)}$ for $\frac{\Phi_{T}^{(\kappa)}}{\Phi_{t}^{(\kappa)}}$. $\Phi_{t, T}^{(\kappa)}$ is called the pricing kernel. To simplify notation in the remainder the index $(\kappa)$ is omitted.

Furthermore, it is well known from Girsanov's theorem that the process $\widetilde{W}$, which is given by

$$
\widetilde{W_{t}}=W_{t}+\int_{0}^{t} \kappa_{u} d u, \quad 0 \leqslant t \leqslant T,
$$

follows a standard Brownian motion on the space $(\Omega, \mathcal{G}, \widetilde{P})$.

Thus, technically the transformation is done by multiplying the asset price under the probability measure $P$ with a suitable $P$-martingale. ${ }^{5}$ To get some economic intuition we apply these results in a simplified economy.

\subsection{Interpretation in a simplified economy}

Consider the following market with no arbitrage opportunities: On the filtered probability space $\left(\Omega^{\prime}, \mathcal{G}^{\prime}, \mathcal{G}_{t}^{\prime}, P^{\prime}\right)$ with a one-dimensional Brownian motion $W^{\prime}$ defined on it the forward price is given by

$$
\begin{aligned}
d F_{t} & =F_{t} \mu_{t} d t+F_{t} \Sigma_{t} d W_{t}^{\prime}, \quad 0 \leqslant t \leqslant T \\
F_{0} & =F_{0}>0
\end{aligned}
$$

with $\mu, \Sigma$ deterministic functions depending on $t$ and $F_{t}$ and $\Sigma \neq 0$. Thus, this market is complete and there is a unique martingale measure. With $\kappa:=\frac{\mu}{\Sigma}$ define $\Phi_{t}=\exp \left(-\int_{0}^{t} \kappa_{u} d W_{u}^{\prime}-\frac{1}{2} \int_{0}^{t}\left|\kappa_{u}\right|^{2} d u\right)$, then applying Itô's formula it is easily seen that $\Phi_{t} F_{t}$ is a martingale and thus

$$
F_{t}=E^{\widehat{P^{\prime}}}\left(F_{T} \mid \mathcal{G}_{t}^{\prime}\right), \quad 0 \leqslant t \leqslant T,
$$

where $\widehat{P^{\prime}}$ is defined by

$$
\frac{d \widehat{P^{\prime}}}{d P^{\prime}}=\Phi_{t}, \quad 0 \leqslant t \leqslant T .
$$

The instantaneous covariance, i.e. the quadratic variation, between this process $\Phi$ and the forward price process $F$ is equal to the instantaneous drift $\mu$ of the forward price process.

\footnotetext{
[25].

${ }^{5}$ For a detailed derivation see for example Karatzas, Shreve [21] and Musiela, Rutkowski
} 
In the remainder of this section we will now briefly discuss the relationship between the general pricing kernel which is based on the no arbitrage assumption and the pricing kernel in a representative investor economy. We assume that the representative investor has a state-independent utility function $U$ of wealth $F_{T}$, which belongs to the set of twice continuous differentiable, strictly increasing and strictly concave functions defined on $(0, \infty)$. We assume that $F$ is given by a stochastic differential equation of form (6). It is well known, that in equilibrium in such an economy, the following equation must hold ${ }^{6}$

$$
\Phi_{0, T}=\frac{\frac{\partial}{\partial x} U\left(F_{T}\right)}{a},
$$

for some scalar $a>0$. Since $E^{P^{\prime}}\left(\Phi_{0, T}\right)=1$ we get $a=E^{P^{\prime}}\left(\frac{\partial}{\partial x} U\left(F_{T}\right)\right)$. Thus, for $\Phi_{t, T}$ we get the common characterization (see for example Bick [2])

$$
\Phi_{t, T}=\frac{\frac{\partial}{\partial x} U\left(F_{T}\right)}{E^{P^{\prime}}\left(\frac{\partial}{\partial x} U\left(F_{T}\right) \mid \mathcal{G}_{t}^{\prime}\right)}, \quad 0 \leqslant t \leqslant T .
$$

Since $\Phi_{t}=\Phi_{0, t}=E^{P^{\prime}}\left(\Phi_{0, T} \mid \mathcal{G}_{t}^{\prime}\right)$ the process $\Phi$ can be characterized by a function $h$ satisfying the Feynman-Kac partial differential equation

$$
\begin{aligned}
0 & =\frac{\partial h}{\partial t}+\frac{\partial h}{\partial x} \mu x+\frac{1}{2} \frac{\partial^{2} h}{\partial x^{2}} \Sigma^{2} x^{2} \\
h(T, x) & =\frac{\frac{\partial}{\partial x} U(x)}{a}
\end{aligned}
$$

by $\Phi_{0, t}=h\left(t, F_{t}\right)$. We derive the following stochastic differential equation for the pricing kernel by applying Itô's formula

$$
\begin{aligned}
d \Phi_{0, t} & =\frac{\partial}{\partial x} h\left(t, F_{t}\right) \Sigma_{t} F_{t} d W_{t}^{\prime}, \quad 0 \leq t \leq T \\
\Phi_{0,0} & =1 .
\end{aligned}
$$

\footnotetext{
${ }^{6}$ See for example Pham, Touzi [27] or Decamps, Lazrak [6].
} 
To derive the elasticity of the pricing kernel we compare this to

$$
\begin{aligned}
d \Phi_{0, t} & =-\Phi_{0, t} \kappa_{t} d W_{t}^{\prime}, \quad 0 \leq t \leq T \\
\Phi_{0,0} & =1
\end{aligned}
$$

and yield

$$
\frac{\kappa_{t}}{\Sigma_{t}}=-\frac{\frac{\partial}{\partial x} h\left(t, F_{t}\right)}{h\left(t, F_{t}\right)} F_{t}, \quad 0 \leq t \leq T .
$$

Equation (8) shows that in the economy under consideration the ratio of the instantaneous volatility of the Girsanov-process, i.e. the market price of risk, and the instantaneous volatility of the asset are equal to the elasticity of the pricing kernel. In $T$ the following relation holds

$$
\left.\frac{\kappa_{t}}{\Sigma_{t}}\right|_{t=T}=-\frac{\frac{\partial}{\partial x} h\left(T, F_{T}\right)}{h\left(T, F_{T}\right)} F_{T}=-\frac{\frac{\partial^{2}}{\partial x^{2}} U\left(F_{T}\right)}{\frac{\partial}{\partial x} U\left(F_{T}\right)} F_{T},
$$

thus the realtive risk aversion of the representative agent equals the elasticity of the pricing kernel. ${ }^{7}$

In the remainder of this paper we will not rely on the existence of an equilibrium. Instead we will only make the assumption that no arbitrage possibilities exist and thus that the transformation of measure is given by an appropriate Girsanov-functional. In this more general case, the pricing kernel is not necessarily a deterministic function of the asset price. ${ }^{8}$

\section{Derivation of Asset Price Processes}

In this section we derive the forward price process of the asset in the market defined in section 3 . The point of start is

$$
F_{t}=E^{P}\left(\Phi_{t, T} F_{T} \mid \sigma\left\{I_{u} \mid 0 \leq u \leq t\right\}\right), \quad 0 \leqslant t \leqslant T,
$$

\footnotetext{
${ }^{7}$ For a more detailed derivation and discussion see for example Decamps, Lazrak [6] and Franke, Stapleton, Subrahmanyam [12]. For a derivation and discussion of the pricing kernel in an equilibrium model not relying on the representative agent assumption see for example Franke, Stapleton, Subrahmanyam [11].

${ }^{8}$ See for example Decamps, Lazrak [6].
} 
where the filtration is the one generated by the information process $I$. Because of the equality of $F_{T}$ and $I_{T}$ the following relationship holds.

$$
F_{t}=E^{\widetilde{P}}\left[F_{T} \mid \sigma\left\{I_{u} \mid 0 \leq u \leq t\right\}\right]=E^{\widetilde{P}}\left[I_{T} \mid \sigma\left\{I_{u} \mid 0 \leq u \leq t\right\}\right], \quad 0 \leqslant t \leqslant T .
$$

We assume that the transformation from $P$ to $\widetilde{P}$ is given by a Girsanovfunctional. More precisely we assume that there is an adapted $\mathbf{R}^{2}$-valued process $\lambda_{s}=\left(\lambda_{s}^{(1)}, \lambda_{s}^{(2)}\right)^{\prime}$ which defines the martingale $(\|\cdot\|$ is the euclidean $\mathbf{R}^{2}$-norm)

$$
\begin{aligned}
\Phi_{0, t}= & \exp \left(-\int_{0}^{t} \lambda_{s}^{(1)} d W_{s}^{I}-\int_{0}^{t} \lambda_{s}^{(2)} d W_{s}^{V}-\frac{1}{2} \int_{0}^{t}\left\|\lambda_{s}\right\|^{2} d s\right), \\
& 0 \leqslant t \leqslant T,
\end{aligned}
$$

and the transformed probability measure

$$
\widetilde{P}(A)=E\left[\Phi_{0, T} 1_{A}\right], \quad A \in \mathcal{F}_{T} .
$$

With this definition $P$ and $\widetilde{P}$ are mutually absolutely continuous on $\mathcal{F}_{T}$ and the process

$$
\left(\begin{array}{c}
\widetilde{W_{t}^{I}} \\
\widetilde{W_{t}^{V}}
\end{array}\right):=\left(\begin{array}{c}
W_{t}^{I}+\int_{0}^{t} \lambda_{s}^{(1)} d s \\
W_{t}^{V}+\int_{0}^{t} \lambda_{s}^{(2)} d s
\end{array}\right), \quad 0 \leqslant t \leqslant T
$$

is a 2-dimensional Brownian motion under $\widetilde{P}$. Hence, we have the representation for $F$ under the probability measure $P$

$$
F_{t}=I_{T}-\int_{t}^{T} \lambda_{s}^{(1)} Z_{s}^{(1)} d s-\int_{t}^{T} \lambda_{s}^{(2)} Z_{s}^{(2)} d s-\int_{t}^{T} Z_{s}^{(1)} d W_{s}^{I}-\int_{t}^{T} Z_{s}^{(2)} d W_{s}^{V}
$$

for $0 \leqslant t \leqslant T$, where $Z=\left(Z^{(1)}, Z^{(2)}\right)^{\prime}$ is the process given by application of the martingale representation theorem on $F$. We assume that $\lambda$ is a smooth deterministic function that may depend on $t, I_{t}$ and $\sigma_{t}^{I}: \lambda\left(t, I_{t}, \sigma_{t}^{I}\right)$.

In the following theorem we give a formula for the forward price $F_{t}$ in terms of the information process and the market price of risk $\lambda\left(t, I_{t}, \sigma_{t}^{I}\right)$. For the derivation we have to solve a forward-backward stochastic differential equation. This is done by application of mathematical theorems given in Ma, Protter, Yong [23] and Ma, Yong [24]. 
Theorem 1 Assume that the information process $I_{t}$ is governed by the stochastic differential equation

$$
I_{t}=I_{0}+\int_{0}^{t} I_{s} \sigma_{s}^{I} d W_{s}^{I}, \quad 0 \leqslant t \leqslant T
$$

where the volatility process $\sigma_{t}^{I}$ is given by

$$
\sigma_{t}^{I}=\sigma_{0}^{I}+\int_{0}^{t} b\left(s, I_{s}, \sigma_{s}^{I}\right) d s+\int_{0}^{t} \sigma^{V}\left(s, I_{s}, \sigma_{s}^{I}\right) d W_{s}^{V}, \quad 0 \leqslant t \leqslant T,
$$

with deterministic smooth functions $b$ and $\sigma^{V}$. Then the forward price $F_{t}$ of the asset admits the following representation under the probability measure $P$

$$
\begin{array}{r}
F_{t}=I_{T}-\int_{t}^{T} \lambda^{(1)}\left(s, I_{s,} \sigma_{s}^{I}\right) Z_{s}^{(1)} d s-\int_{t}^{T} \lambda^{(2)}\left(s, I_{s,} \sigma_{s}^{I}\right) Z_{s}^{(2)} d s \\
-\int_{t}^{T} Z_{s}^{(1)} d W_{s}^{I}-\int_{t}^{T} Z_{s}^{(2)} d W_{s}^{V}, \\
0 \leqslant t \leqslant T,
\end{array}
$$

with

$$
Z_{t}=\left(\begin{array}{c}
Z_{t}^{(1)} \\
Z_{t}^{(2)}
\end{array}\right)=\left(\begin{array}{cc}
I_{t} \sigma_{t}^{I} & 0 \\
0 & \sigma^{V}\left(t, I_{t}, \sigma_{t}^{I}\right)
\end{array}\right) \nabla u\left(t, I_{t}, \sigma_{t}^{I}\right), \quad 0 \leqslant t \leqslant T,
$$

and

$$
\nabla u(t, x)=\left(u_{x_{1}}(t, x), u_{x_{2}}(t, x)\right)^{T},
$$

where $u:[0, T] \times \mathbf{R}^{2} \rightarrow \mathbf{R}$ is the solution of the partial differential equation

$$
\begin{array}{r}
0=u_{t}\left(t, x_{1}, x_{2}\right)-\lambda^{(1)}\left(t, x_{1}, x_{2}\right) x_{1} x_{2} u_{x_{1}}\left(t, x_{1}, x_{2}\right) \\
-\lambda^{(2)}\left(t, x_{1}, x_{2}\right) \sigma^{V}\left(t, x_{1}, x_{2}\right) u_{x_{2}}\left(t, x_{1}, x_{2}\right) \\
+\frac{1}{2}\left(x_{1}^{2} x_{2}^{2} u_{x_{1} x_{1}}\left(t, x_{1}, x_{2}\right)+\left(\sigma^{V}\left(t, x_{1}, x_{2}\right)\right)^{2} u_{x_{2} x_{2}}\left(t, x_{1}, x_{2}\right)\right) \\
+b\left(t, x_{1}, x_{2}\right) u_{x_{2}}\left(t, x_{1}, x_{2}\right) \\
u\left(T, x_{1}, x_{2}\right)=x_{1}
\end{array}
$$

for $0 \leqslant t \leqslant T$, with indices on the function $u$ indicating partial derivatives. Moreover $F_{t}$ is given by

$$
F_{t}=u\left(t, I_{t}, \sigma_{t}^{I}\right), \quad 0 \leqslant t \leqslant T .
$$


Proof. We have the following system:

the forward stochastic differential equation $(F S D E)$ for the information process $I_{t}$ and its volatility process $\sigma_{t}^{I}$

$$
\begin{aligned}
\left(\begin{array}{c}
I_{t} \\
\sigma_{t}^{I}
\end{array}\right)= & \left(\begin{array}{c}
X_{t}^{(1)} \\
X_{t}^{(2)}
\end{array}\right)=\left(\begin{array}{c}
X_{0}^{(1)} \\
X_{0}^{(2)}
\end{array}\right)+\int_{0}^{t}\left(\begin{array}{c}
0 \\
b\left(s, X_{s}^{1}, X_{s}^{2}\right)
\end{array}\right) d s \\
& +\int_{0}^{t} \underbrace{\left(\begin{array}{cc}
X_{s}^{(1)} X_{s}^{(2)} & 0 \\
0 & \sigma^{V}\left(s, X_{s}^{(1)}, X_{s}^{(2)}\right)
\end{array}\right)}_{\sigma}\left(\begin{array}{c}
d W_{s}^{I} \\
d W_{s}^{V}
\end{array}\right), \quad 0 \leqslant t \leqslant T,
\end{aligned}
$$

and the backward stochastic differential equation $(B S D E)$ for the forward price $F_{t}$ of the asset

$$
\begin{aligned}
F_{t}= & Y_{t}=X_{T}^{1}-\int_{t}^{T}\left(\lambda^{(1)}\left(s, X_{s}^{(1)}, X_{s}^{(2)}\right) Z_{s}^{(1)}+\lambda^{(2)}\left(s, X_{s}^{(1)}, X_{s}^{(2)}\right) Z_{s}^{(2)}\right) d s \\
& -\int_{t}^{T}\left(\begin{array}{c}
Z_{s}^{(1)} \\
Z_{s}^{(2)}
\end{array}\right)^{T}\left(\begin{array}{c}
d W_{s}^{I} \\
d W_{s}^{V}
\end{array}\right), \quad 0 \leqslant t \leqslant T .
\end{aligned}
$$

The coupled system (11) and (12) is a forward-backward stochastic differential equation (FBSDE). We use the Four-Step-Scheme given in Ma, Protter, Yong [23], more precisely we apply the version given in Ma, Yong [24], to find the solution.

Step 1: We define the function $z(t, x, y, w)=\sigma^{T}(t, x, y) w$ for $(t, x, y, w) \in$ $\mathbf{R} \times \mathbf{R}^{2} \times \mathbf{R} \times \mathbf{R}^{2}$. With this definition we have the 2-dimensional function

$$
z(t, x, y, w)=\left(\begin{array}{l}
z_{1} \\
z_{2}
\end{array}\right)(t, x, y, w)=\left(\begin{array}{c}
x_{1} x_{2} w_{1} \\
\sigma^{V}\left(t, x_{1}, x_{2}\right) w_{2}
\end{array}\right) .
$$

Step 2: With the function $z$ we solve the partial differential equation (PDE)

$$
\begin{aligned}
u(t, x)= & x_{1}+\int_{t}^{T}-\lambda^{(1)}\left(s, x_{1}, x_{2}\right) z_{1}(s, x, u(s, x), \nabla u(s, x)) \\
& -\lambda^{(2)}\left(s, x_{1}, x_{2}\right) z_{2}(s, x, u(s, x), \nabla u(s, x)) \\
& +\frac{1}{2} \operatorname{tr}\left\{\left(\begin{array}{cc}
x_{1} x_{2} & 0 \\
0 & \sigma^{V}(s, x)
\end{array}\right)^{2}\left(\begin{array}{cc}
u_{x_{1} x_{1}} & u_{x_{1} x_{2}} \\
u_{x_{2} x_{1}} & u_{x_{2} x_{2}}
\end{array}\right)(s, x)\right\} \\
& +\left\langle\left(\begin{array}{c}
0 \\
b(s, x)
\end{array}\right),\left(\begin{array}{l}
u_{x_{1}}(s, x) \\
u_{x_{2}}(s, x)
\end{array}\right)\right\rangle d s, \quad 0 \leqslant t \leqslant T
\end{aligned}
$$


where by $\operatorname{tr}\{\cdot\}$ we denote the trace of a $2 \times 2$-matrix and by $\langle\cdot, \cdot\rangle$ the inner product of the Euclidean space $\mathbf{R}^{2}$. Hence, we have for $0 \leqslant t \leqslant T$

$$
\begin{aligned}
0= & u_{t}(t, x)-\lambda^{(1)}(t, x) x_{1} x_{2} u_{x_{1}}(t, x)-\lambda^{(2)}(t, x) \sigma^{V}(t, x) u_{x_{2}}(t, x) \\
& +\frac{1}{2}\left(x_{1}^{2} x_{2}^{2} u_{x_{1} x_{1}}(t, x)+\left(\sigma^{V}(t, x)\right)^{2} u_{x_{2} x_{2}}(t, x)\right)+b(t, x) u_{x_{2}}(t, x) \\
u(T, x)= & x_{1} .
\end{aligned}
$$

We jump directly to Step 4, omitting Step 3: We define $Y_{t}=u\left(t, X_{t}\right)$ and $Z_{t}=\sigma^{T}\left(t, X_{t}, u\left(t, X_{t}\right)\right) \nabla u\left(t, X_{t}\right)$, then $(X, Y, Z)$ is an adapted solution of (11) and (12).

Theorem 1 shows the close relationship between the asset price process and the information process. Given the information process and the pricing kernel Theorem 1 establishes a characterization of the asset price process as a function of the information process and the pricing kernel. The drift of the asset price process is governed by the market price of risk $\lambda_{t}$ and the diffusion of the asset price process $Z_{t}$. The diffusion $Z_{t}$ depends on the information process $I_{t}$ itself, on the volatility process of $I_{t}$, i.e. $\sigma_{t}^{I}$, and on the first derivatives w.r.t. $x_{1}$ and $x_{2}$ of the function characterizing the asset price: $\frac{\partial u}{\partial x_{1}}\left(t, I_{t}, \sigma_{t}^{I}\right), \frac{\partial u}{\partial x_{2}}\left(t, I_{t}, \sigma_{t}^{I}\right)$. Thus, with Theorem 1 we have an explicit representation of the asset price process in terms of the information process and the pricing kernel for a 2-dimensional market model. The application of Theorem 1 to $n$-dimensional market models is straight forward.

It is obvious from Theorem 1 that the drift of the asset price process depends on the volatility of the information process. Thus, empirical studies implicitly assuming non stochastic volatility of the information process may find unexplainable variations in the drift. Further, neglecting stochastic volatility of the information process leads to only one risk premium in the asset price process, i.e. $\sigma_{t}^{V}=0$ provides $Z_{t}^{(2)}=0$.

To gain some better understanding of the implications of Theorem 1 in the remainder of this section we discuss the case when all the coefficients are only functions in $t$. Thus, with this assumption equation (10) simplifies to

$$
\begin{aligned}
0= & u_{t}\left(t, x_{1}, x_{2}\right)-\lambda^{(1)}(t) x_{1} x_{2} u_{x_{1}}\left(t, x_{1}, x_{2}\right) \\
& -\lambda^{(2)}(t) \sigma^{V}(t) u_{x_{2}}\left(t, x_{1}, x_{2}\right) \\
& +\frac{1}{2}\left(x_{1}^{2} x_{2}^{2} u_{x_{1} x_{1}}\left(t, x_{1}, x_{2}\right)+\left(\sigma^{V}(t)\right)^{2} u_{x_{2} x_{2}}\left(t, x_{1}, x_{2}\right)\right) \\
& +b(t) u_{x_{2}}\left(t, x_{1}, x_{2}\right), \quad 0 \leqslant t \leqslant T, \\
u(T, x)= & x_{1}
\end{aligned}
$$


and the solution of equation (13) for $u(t, x)$ is (see Appendix)

$$
\begin{aligned}
u\left(t, x_{1}, x_{2}\right)= & x_{1} \exp \left(-x_{2} \int_{t}^{T} \lambda^{(1)}(s) d s\right) \\
& \exp \left(\int_{t}^{T}\left(-\lambda^{(2)}(r) \sigma^{V}(r)+b(r)\right)\left(\int_{r}^{T}-\lambda^{(1)}(s) d s\right) d r\right) \\
& \exp \left(\int_{t}^{T} \frac{1}{2}\left(\sigma^{V}(r)\right)^{2}\left(\int_{r}^{T}-\lambda^{(1)}(s) d s\right)^{2} d r\right), \quad 0 \leqslant t \leqslant T .
\end{aligned}
$$

Hence, $F_{t}$ is given by

$$
\begin{aligned}
F_{t}= & I_{t} \exp \left(-\sigma_{t}^{I} \int_{t}^{T} \lambda^{(1)}(s) d s\right) \\
& \exp \left(-\int_{t}^{T}\left(-\lambda^{(2)}(r) \sigma^{V}(r)+b(r)\right)\left(\int_{r}^{T} \lambda^{(1)}(s) d s\right) d r\right) \\
& \exp \left(\int_{t}^{T} \frac{1}{2}\left(\sigma^{V}(r)\right)^{2}\left(\int_{r}^{T} \lambda^{(1)}(s) d s\right)^{2} d r\right), \quad 0 \leqslant t \leqslant T,
\end{aligned}
$$

and

$$
Z_{t}=\left(\begin{array}{c}
Z_{t}^{(1)} \\
Z_{t}^{(2)}
\end{array}\right)=F_{t}\left(\begin{array}{l}
\sigma_{t}^{I} \\
-\sigma^{V}(t) \int_{t}^{T} \lambda^{(1)}(s) d s
\end{array}\right) \quad, 0 \leqslant t \leqslant T .
$$

The forward price of the asset can be rewritten as

$$
\begin{aligned}
d F_{t}= & F_{t} \sigma_{t}^{F}\left(\sqrt{1-\rho_{t}^{2}} \lambda^{(1)}(t)+\lambda^{(2)}(t) \rho_{t}\right) d t \\
& +F_{t} \sigma_{t}^{F}\left(\sqrt{1-\rho_{t}^{2}} d W_{t}^{I}+\rho_{t} d W_{t}^{V}\right), \quad 0 \leqslant t \leqslant T, \\
F_{T}= & I_{T}
\end{aligned}
$$

with

$$
F_{t} \sigma_{t}^{F}:=\sqrt{\left(Z_{t}^{(1)}\right)^{2}+\left(Z_{t}^{(2)}\right)^{2}}
$$

and

$$
\rho_{t}:=\frac{Z_{t}^{(2)}}{\sqrt{\left(Z_{t}^{(1)}\right)^{2}+\left(Z_{t}^{(2)}\right)^{2}}}
$$


$\rho$ is the instantaneous correlation between the asset price and its volatility. Hence, in the usual notation (we make use of $d W_{t}^{F}=\sqrt{1-\rho_{t}^{2}} d W_{t}^{I}+\rho_{t} d W_{t}^{V}$ ) the forward price $F$ is given by

$$
d F_{t}=F_{t} \sigma_{t}^{F}\left(\sqrt{1-\rho_{t}^{2}} \lambda^{(1)}(t)+\lambda^{(2)}(t) \rho_{t}\right) d t+F_{t} \sigma_{t}^{F} d W_{t}^{F}, \quad 0 \leqslant t \leqslant T,
$$

where $W^{F}$ and $W^{V}$ have correlation $\rho$.

It is important to notice that even though the information process and its volatility process are uncorrelated, the asset price process and its volatility process are correlated. This is in contrast to an assumption in many stochastic volatility models (see for example Hull, White [18] or Stein, Stein [31]) and has already been criticized by Pham and Touzi [27]. Corollary 1 establishes conditions for $\rho \equiv 0$.

Corollary 1 The correlation $\rho$ is zero if and only if

(i) $\sigma^{V}=0$

or

(ii) $\lambda^{(1)}=0$ a.s.

Condition $(i)$ is trivial since it implies that the volatility of the information process is not governed by a Brownian motion. Condition (ii) implies that the correlation $\rho$ is zero, if $\lambda^{(1)}$, the risk premium relative to the source of uncertainty $W^{I}$, is zero. Thus Corollary 1 gives an economic foundation for stochastic volatility models where the asset price and its volatility are correlated. ${ }^{9}$

Corollary 2 Assume that the market price of risk $\lambda^{(1)}$ is positive (resp. $\lambda^{(1)}$ negative) and $\sigma^{V}>0$. Then the correlation $\rho$ between the forward price and its volatility is negative (resp. positive).

This result is obvious from equation (15). Ruling out the implausible case that the market price of risk is not positive, we can conclude that the correlation is negative. Thus, our analysis supports the usual result in empirical studies that volatility and price of an asset are negatively correlated. But our argument is inverse to the argument which bases on the leverage effect. The intuition of Corollary 2 is as follows: For a higher volatility $\sigma_{t}^{I}$, hence a higher risk, at time $t$ a risk averse investor requires a higher reward. And

\footnotetext{
${ }^{9}$ See for example Schöbel, Zhu [30] and Heston, Nandi [15].
} 
thus, the current price $F_{t}$ has to be lower, since the terminal value $F_{T}$ of the forward price is given by $I_{T}$ (remember $\left.F_{T}=I_{T}\right) .{ }^{10}$

With equation (16) we can give an explanation for the well documented time pattern of the sharpe ratio $\frac{\mu}{\sigma^{F}}$, with $\mu$ the instantaneous drift of $F$ ( $\mu_{t}=\sigma_{t}^{F}\left(\sqrt{1-\rho_{t}^{2}} \lambda^{(1)}(t)+\lambda^{(2)}(t) \rho_{t}\right)$ for $0 \leqslant t \leqslant T$, defined as usual) :

$$
\frac{\mu_{t}}{\sigma_{t}^{F}}:=\sqrt{1-\rho_{t}^{2}} \lambda^{(1)}(t)+\lambda^{(2)}(t) \rho_{t}, \quad 0 \leqslant t \leqslant T .
$$

This equation shows that the sharpe ratio $\frac{\mu}{\sigma^{F}}$ may be time-varying and stochastic even if the risk premia are constant, i.e. $\lambda^{(1)}(t)=$ const., $\lambda^{(2)}(t)=$ const. in equation (17). Hence, equation (17) suggests that seemingly unexplainable variations of the risk premia $\lambda$ and the sharpe ratio $\frac{\mu}{\sigma^{F}}$ may be due to the assumption of a constant correlation $\rho$ between $W^{F}$ and $W^{V}$.

To illustrate our results we now consider the case when the volatility of the information process depends on time only.

Example Let $\sigma_{t}^{I}$ depend on time only. Then the volatility of the information process satisfies

$$
\sigma_{t}^{I}=\sigma_{0}^{I}+\int_{0}^{t} b(s) d s, \quad 0 \leq t \leq T
$$

Then, by Theorem 1, the forward price of the asset satisfies the stochastic differential equation

$$
d F_{t}=F_{t} \lambda^{(1)}(t) \sigma_{t}^{I} d t+F_{t} \sigma_{t}^{I} d W_{t}^{F}, \quad 0 \leqslant t \leqslant T .
$$

With this simplified model it is easily seen, that the properties of the volatility of the information process transfer to the properties of the drift and the volatility of the asset price process. The drift of the asset price process is equal to the volatility of the information process multiplied by the market price of risk. The volatility of the information process and the volatility of the asset price process are identical.

\footnotetext{
${ }^{10}$ The described effect is known as the volatility feedback effect. For similar results in discrete time see Campbell, Hentschel [3] and Wu [33].
} 
Further simplifying the model by assuming that the volatility of the information process is constant, i.e. the information process is governed by a geometric Brownian motion and assuming $\lambda^{(1)}$ constant, we get

$$
d F_{t}=F_{t} \lambda^{(1)} \sigma^{I} d t+F_{t} \sigma^{I} d W_{t}^{F}
$$

From equation (18) it is obvious that the asset price follows a geometric Brownian motion process if the information process has constant volatility and the risk premium is constant.

In this case the relative risk aversion is constant, too. From

$$
\frac{\lambda^{(1)}}{\sigma^{I}}=-\frac{\frac{\partial^{2}}{\partial x^{2}} U\left(F_{T}\right)}{\frac{\partial}{\partial x} U\left(F_{T}\right)} F_{T}=\text { constant }
$$

we derive the following form of the utility function of the representative investor for the case $\lambda^{(1)} \neq \sigma^{I}$

$$
U(X)=\frac{C_{1} X^{1-\frac{\lambda^{(1)}}{\sigma^{I}}}}{1-\frac{\lambda^{(1)}}{\sigma^{I}}}+C_{2}, \quad X \in \mathbf{R}^{+},
$$

where $C_{1}, C_{2}$ are constants. This utility function belongs to the HARAclass. In contrast, if $\lambda^{(1)}=\sigma^{I}$, we get

$$
U(X)=\bar{C}_{1} \ln (X)+\bar{C}_{2}, \quad X \in \mathbf{R}^{+},
$$

with $\bar{C}_{1}, \bar{C}_{2}$ being two constant parameters. Thus our example illustrates that a forward price process governed by a geometric Brownian motion is consistent with an information process with constant volatility and a representative investor with a utility function given by equation (19) or equation (20) where the relative risk aversion is given by the sharpe ratio divided by the volatility. ${ }^{11}$

\footnotetext{
${ }^{11}$ See also Bick [1], [2] and Franke, Stapleton, Subrahmanyam [12].
} 


\section{Conclusions}

Different approaches to examine asset price processes exist. On the one hand, due to the availability of financial markets data, an enormous amount of time series properties have been well documented by recent empirical studies. On the other hand many theoretical papers establish necessary characteristics of asset price processes to be consistent with an equilibrium. Unfortunately the gap between empirically well documented stylized facts and theoretically explainable facts is still vast. The purpose of this paper was on the one hand to introduce new mathematical tools for the analysis of asset price processes and to give a general context in which the behavior of asset price processes can be studied. On the other hand we wanted to show that the discrepancy between empirical and theoretical findings may result from the fact that one important source of risk is neglected. In this paper we have considered this risk, i.e. that even the risk of an asset is unknown and therefore risky, too. In this case the information process has stochastic volatility. With this generalization of usual information processes a foundation for stochastic volatility models of asset prices has been established. Further it has been shown that the still prevalent assumption of zero correlation between asset prices and their volatility is not sensible and hence we gave a foundation for option pricing models allowing for correlation between asset prices and their volatility. Further, we have shown that under resonable assumptions the correlation between asset prices and their volatility is negative. Finally we have shown the close relationship between the volatility process of the information process and the risk premia of an asset. Because of this dependence of the risk premia on the volatility process a theoretical foundation can be given for financial market phenomena.

Our approach offers numerous avenues for future research. More research should be devoted to the information process and its volatility. Because of the established coherence between information processes and asset price processes this is no more a purely theoretical task. Characteristics of information processes can be deduced from asset price processes. Hence, it is possible to investigate empirically whether asset price processes are consistent with the strong assumptions usually made on information processes. 


\section{Acknowledgement}

We are grateful for extensive help by Günter Franke and Michael Kohlmann. We strongly benefited also from conversations with Günther Bamberg, Jan Beran, Jürgen Eichberger, Yuanhua Feng, Frank Gerhard, Stamen Gortchev, Joachim Grammig, Dieter Hess, Johannes Leitner, Christian R. Meurer, Rainer Schöbel, Jack Wahl and Jochen Wilhelm. An earlier version (On the Relationship of Information Processes and Asset Price Processes) has been presented at the Annual Congress of the European Economic Association in Bolzano, the Annual Congress of the Verein für Socialpolitik in Berlin, the Annual Congress of the Deutsche Mathematiker-Vereinigung (DMV) in Dresden and the Annual Congress of the German Finance Association in Konstanz. We are grateful to the participants for useful comments.

Financial support by the Center of Finance and Econometrics and the Zentrum für Europäische Wirtschaftsforschung is gratefully acknowledged. Erik Lüders appreciates a grant by the Deutsche Bundesbank.

\section{A Appendix}

The PDE, with indices on the functions indicating partial derivatives,

$$
\begin{aligned}
0= & u_{t}\left(t, x_{1}, x_{2}\right)-\lambda^{(1)}\left(t, x_{1}, x_{2}\right) x_{1} x_{2} u_{x_{1}}\left(t, x_{1}, x_{2}\right) \\
& -\lambda^{(2)}\left(t, x_{1}, x_{2}\right) \sigma^{V}\left(t, x_{1}, x_{2}\right) u_{x_{2}}\left(t, x_{1}, x_{2}\right) \\
& +\frac{1}{2}\left(x_{1}^{2} x_{2}^{2} u_{x_{1} x_{1}}\left(t, x_{1}, x_{2}\right)+\left(\sigma^{V}\left(t, x_{1}, x_{2}\right)\right)^{2} u_{x_{2} x_{2}}\left(t, x_{1}, x_{2}\right)\right) \\
& +b\left(t, x_{1}, x_{2}\right) u_{x_{2}}\left(t, x_{1}, x_{2}\right) \\
u(T, x)= & x_{1}
\end{aligned}
$$

can be solved for only time-dependent coefficients.

For notational simplicity define the functions: $\alpha:=-\lambda^{1}, \beta:=-\lambda^{2} \sigma^{V}+b$, $\gamma:=\sigma^{V}=-\frac{\beta-b}{\lambda^{2}}$. Hence, the PDE can be written as

$$
\begin{aligned}
0= & u_{t}\left(t, x_{1}, x_{2}\right)+\alpha\left(t, x_{1}, x_{2}\right) x_{1} x_{2} u_{x_{1}}\left(t, x_{1}, x_{2}\right)+\beta\left(t, x_{1}, x_{2}\right) u_{x_{2}}\left(t, x_{1}, x_{2}\right) \\
& +\frac{1}{2} x_{1}^{2} x_{2}^{2} u_{x_{1} x_{1}}\left(t, x_{1}, x_{2}\right)+\frac{1}{2} \gamma^{2}\left(t, x_{1}, x_{2}\right) u_{x_{2} x_{2}}\left(t, x_{1}, x_{2}\right)
\end{aligned}
$$

with the boundary condition $u(T, x)=x_{1}$. 
We have to consider the two cases $x_{1}=0$ and $x_{1} \neq 0$. If $x_{1}=0$ we have the trivial solution. Thus, in the following we choose $x_{1} \neq 0$.

Assume that the coefficients of the PDE do not depend on $x_{1}$. Then $u$ can be separated as follows: $u\left(t, x_{1}, x_{2}\right)=\varphi\left(x_{1}, x_{2}\right) \psi\left(t, x_{2}\right)$. With the boundary condition $u\left(T, x_{1}, x_{2}\right)=x_{1}$ it follows $\varphi\left(x_{1}, x_{2}\right) \psi\left(T, x_{2}\right)=x_{1}$ and because of $x_{1} \neq 0: \psi\left(T, x_{2}\right) \neq 0$ for all $x_{2}$. Hence, the following relationship holds: $\varphi\left(x_{1}, x_{2}\right)=\frac{x_{1}}{\psi\left(T, x_{2}\right)}$.

After some computation the partial differential equation can be written as (without noting the variables of the functions $\alpha, \beta$ and $\gamma$ )

$$
\begin{aligned}
0= & \psi_{t}\left(t, x_{2}\right)+\psi\left(t, x_{2}\right)\left(\alpha x_{2}-\beta \frac{\psi_{x_{2}}\left(T, x_{2}\right)}{\psi\left(T, x_{2}\right)}+\gamma^{2} \frac{\psi_{x_{2}}^{2}\left(T, x_{2}\right)}{\psi^{2}\left(T, x_{2}\right)}-\frac{\gamma^{2}}{2} \frac{\psi_{x_{2} x_{2}}\left(T, x_{2}\right)}{\psi\left(T, x_{2}\right)}\right) \\
& +\psi_{x_{2}}\left(t, x_{2}\right)\left(\beta-\gamma^{2} \frac{\psi_{x_{2}}\left(T, x_{2}\right)}{\psi\left(T, x_{2}\right)}\right)+\frac{\gamma^{2}}{2} \psi_{x_{2} x_{2}}\left(t, x_{2}\right),
\end{aligned}
$$

without loss of generality choose the boundary condition $\psi\left(T, x_{2}\right)=1$.

Now assume that all coefficients are only time-dependent.

In the case of constant volatility, that is $\sigma^{V} \equiv 0$ and $b \equiv 0$ and hence $\beta \equiv 0$ and $\gamma \equiv 0$, we have the following solution of the PDE

$$
\psi\left(t, x_{2}\right)=\exp \left(x_{2} \int_{t}^{T} \alpha(s) d s\right) .
$$

With this knowledge, for the general PDE with time-dependent coefficients we try the ansatz

$$
\psi\left(t, x_{2}\right)=\exp \left(-x_{2} \int_{0}^{t} \alpha(s) d s\right) A(t) .
$$

with $A(T)=1$. This leads to an ordinary first-order differential equation for $A$ which has the solution

$$
A(t)=\exp \left(\frac{1}{2} \int_{t}^{T} 2 \beta(r)\left(\int_{r}^{T} \alpha(s) d s\right)+\gamma^{2}(r)\left(\int_{r}^{T} \alpha(s) d s\right)^{2} d r\right) .
$$

Hence, the solution of the PDE for $u$ is

$$
\begin{aligned}
u\left(t, x_{1}, x_{2}\right)= & x_{1} \exp \left(x_{2} \int_{t}^{T} \alpha(s) d s\right) \\
& \exp \left(\frac{1}{2} \int_{t}^{T} 2 \beta(r)\left(\int_{r}^{T} \alpha(s) d s\right)+\gamma^{2}(r)\left(\int_{r}^{T} \alpha(s) d s\right)^{2} d r\right)
\end{aligned}
$$


and in terms of the original coefficients

$$
\begin{aligned}
u\left(t, x_{1}, x_{2}\right)= & x_{1} \exp \left(-x_{2} \int_{t}^{T} \lambda^{1}(s) d s\right) \\
& \exp \left(\int_{t}^{T}\left(-\lambda^{(2)}(r) \sigma^{V}(r)+b(r)\right)\left(-\int_{r}^{T} \lambda^{(1)}(s) d s\right) d r\right) \\
& \exp \left(\int_{t}^{T} \frac{1}{2}\left(\sigma^{V}(r)\right)^{2}\left(-\int_{r}^{T} \lambda^{(1)}(s) d s\right)^{2} d r\right) .
\end{aligned}
$$




\section{References}

[1] Bick, Avi (1987), On the Consistency of the Black-Scholes Model with a General Equilibrium Framework, Journal of Financial and Quantitative Analysis 22, 259-275.

[2] Bick, Avi (1990), On Viable Diffusion Price Processes of the Market Portfolio; Journal of Finance 45, 673-689.

[3] Campbell, John Y. and Hentschel, Ludger (1992), No news is good news: An Asymmetric Model of Changing Volatility in Stock Returns, Journal of Financial Economics, 31, 281-318.

[4] Canina, Linda and Figlewski, Stephen (1993), The Informational Content of Implied Volatility, Review of Financial Studies, Vol. 6, No. 3, 659-681.

[5] David, Alexander (1997), Fluctuating Confidence in Stock Markets: Implications for Returns and Volatility, Journal of Financial and Quantitative Analysis, 32 (4), 427-462.

[6] Decamps, J. P. and Lazrak A. (2000), A Martingale Characterization of Equilibrium Asset Price Processes, Economic Theory 15 (1), 207-213.

[7] Ederington, Louis H. and Lee, Jae Ha (1993), How Markets Process Information: News Releases and Volatility, Journal of Finance 48 (4).

[8] El Karoui, N.; Peng, S. and Quenez, M. C. (1997), Backward stochastic differential equations in finance, Mathematical Finance, Vol.7, No.1, 171.

[9] Fama, Eugene F. and French, Kenneth R. (1988), Permanent and Temporary Components of Stock Prices, Journal of Political Economy 96 (2), 246-73.

[10] Föllmer, Hans and Schweizer, Martin (1991), Hedging of Contingent Claims under Incomplete Information, Applied Stochastic Analysis, Vol. 5, 389-414.

[11] Franke, Günter; Stapleton, Richard C. and Subrahmanyam, Marti G. (1998), Who Buys and Who Sells Options, The Role of Options in an 
Economy with Background Risk, Journal of Economic Theory 82, 89109.

[12] Franke, Günter; Stapleton, Richard C. and Subrahmanyam, Marti G. (1999), When are Options Overpriced? The Black-Scholes Model and Alternative Characterisations of the Pricing Kernel, European Finance Review 3(1), 79-102.

[13] Ghysels, Eric; Harvey, Andrew C. and Renault, Eric (1996), Stochastic Volatility, in: Handbook of Statistics, Vol. 14, 119-191.

[14] He, Hua and Leland, Hayne (1993), On Equilibrium Asset Price Processes, Review of Financial Studies, Vol. 6, No. 3, 593-617.

[15] Heston, S. L.; Nandi S. (1997), A Closed-Form GARCH Option Pricing Model, Working Paper 97-9, Federal Reserve Bank of Atlanta.

[16] Hodges, Stewart and Selby, Michael (1997), The Risk Premium in Trading Equilibria which Support Black-Scholes Option Pricing, in Michael Dempster, Stanley Pliska (ed.) Mathematics of Derivative Securities, Cambridge University Press.

[17] Hodges, Stewart and Carverhill, Andrew (1993), Quasi Mean Reversion in an Efficient Stock Market: The Characterisation of Economic Equilibria which Support Black-Scholes Option Pricing, Economic Journal 103, 395-405.

[18] Hull, John C. and White, Alan (1987), The pricing of Options with Stochastic Volatilities, Journal of Finance 42, 281-300.

[19] Huang, Chi-Fu (1985), Information Structure and Equilibrium Asset Prices, Journal of Economic Theory 35, 33-71.

[20] Jackwerth, Jens Carsten and Rubistein, Mark (1996), Recovering Probability Distributions from Contemporaneous Security Prices, Journal of Finance, 51, 1611-1631.

[21] Karatzas, Ioannis and Shreve, Steven E. (1999), Brownian Motion and Stochastic Calculus, Second Edition, Springer.

[22] Karatzas, Ioannis and Shreve, Steven E. (1998), Methods of Mathematical Finance, Springer. 
[23] Ma, Jin ; Protter, Philip and Yong, Jiongmin (1994), Solving forwardbackward stochastic differential equations explicitly - a four step scheme, Probab. Theory Relat. Fields 98, 339-359.

[24] Ma, Jin and Yong, Jiongmin (1997), Adapted solution of a degenerate backward spde, with applications, Stochastic Processes and their Applications 70, 59-84.

[25] Musiela, Marek and Rutkowski, Marek (1997), Martingale Methods in Financial Modelling, Springer.

[26] Pardoux, E. and Peng, S. (1992), Backward Stochastic Differential Equations and Quasilinear Parabolic Partial Differential Equations. In: Rozovskii, B. L. and Sowers, R. S. (eds), Stochastic partial differential equations and their applications (Lect. Notes Control inf. Sci. 176, pp. 200-217) Springer.

[27] Pham, Huyên and Touzi, Nizar (1996), Equilibrium State Prices in a Stochastic Volatility Model, Mathematical Finance, Vol.6, No.2 , 215236.

[28] Poterba, James and Summmers, Lawrence (1988), Mean Reversion in Stock Prices, Journal of Financial Economics 22 (1), 27-59.

[29] Rubinstein, Mark (1994), Implied Binomial Trees, Journal of Finance, 49, 771-818.

[30] Schöbel, Rainer; Zhu, Jianwei (1999), Stochastic Volatility With an Ornstein-Uhlenbeck Process: An Extension, European Finance Review $3,23-46$.

[31] Stein, Elias M. and Stein, Jeremy C.(1991), Stock Price Distributions with stochastic Volatility: An Analytic Approach, Review of Financial Studies, Vol. 4, No. 4, 727-752.

[32] Veronesi, Pietro (2000), How Does Information Quality Affect Stock Returns? Journal of Finance 55 (2), 807-837.

[33] Wu, Guojun (2000), The Determinants of Asymmetric Volatility, forthcoming: Review of Financial Studies. 